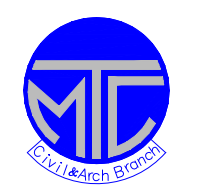

ICCAE
Military Technical College

Kobry Elkobbah, Cairo, Egypt 7hㅡ International Conference On Civil \& Architecture

Engineering

\title{
NUMERICAL AND EXPERIMENTAL INVESTIGATIONS INTO THE INTERACTION BETWEEN SURFACE FOOTINGS AND SHALLOW TUNNELS
}

\author{
Mostafa MOHAMED $^{1}$, and Nabil NAGY ${ }^{2}$
}

\begin{abstract}
Tunnels are constructed to resolve sewerage and transportation problems and may pass under or near existing buildings. Construction of shallow tunnels in urban areas can result in significant ground movement and could damage the function of civil engineering structures if care is not undertaken. On the other hand, building of structures above existing tunnels could lead to an increase in the stresses within the soil mass, which in turn could increase stresses transferred to and deformation of the tunnel lining. The interaction between the surface footings and underground tunnels depends upon several factors including the relative dimensions of the surface footings and tunnel as well as depth and eccentricity of the proposed tunnel. This paper presents the results of numerical and experimental investigations undertaken to explore the interaction between surface loading and underground tunnels. Experimental results indicated that the deformation of the tunnel lining is significantly influenced by the pressure exerted on the surface footings. A finite element code "ABAQUS" is utilised to simulate the interaction between a surface footing and an underground tunnel. Results of the numerical simulations seem along the lines of the experimental ones.
\end{abstract}

Keywords: Bearing capacity, Footing-tunnel interaction, Numerical modelling, Physical tests

\footnotetext{
${ }^{1}$ Lecturer in Geotechnical Engineering, School of Engineering, Design and Technology, University of Bradford, Bradford, UK. Email: m.h.a.mohamed@bradford.ac.uk

${ }^{2}$ Lecturer in Geotechnical Engineering Civil Eng. Department, Military Technical Collage Cairo, EGYPT , nabilmnagy@yahoo.com
} 


\section{INTRODUCTION}

Underground structures such as tunnels, pipes and shelters are essential elements resolving congestion problems, conveying sewage to treatment plants and protecting public from terrorist attacks. Due to increasing urbanisation, these structures might pass/be found under or near existing buildings. For example shallow underground structures built in developed areas could result in a significant ground movement if care is not undertaken. Another scenario is when a structure is built above an existing underground structure. In this case the underground structure may suffer from increased pressure, which might cause some distress within the lining and lead to excessive deformation. Thus, it is imperative that improved knowledge is acquired for the interaction between surface loads and underground structures.

The theory of bearing capacity of soils is originally developed for a strip footing on homogeneous sand and clay soils (Terzaghi, 1943) and is then extended to reflect many factors including the shape of footing and loading condition (see for example, Meyerhof, 1963). The theory of bearing capacity can not be applied if the subsurface soils contain voids or tunnels. Unsupported underground voids could occur naturally due to for example solution cavities. Wang and Baus (1980) investigated the settlement behaviour of continuous footings located on a subsurface soil containing a void. Their study indicated that the settlement of footings is dependent upon the location and size of the underground void. However, if the void exists below a certain depth which is often called the critical depth, the presence of the underground void has a negligible effect on the bearing capacity and associated settlement (Baus and Wang, 1983). This was further elaborated by Wood and Lamach (1984) who concluded that if the void is located within what is called the critical zone, there will be a reduction in the bearing capacity as well as increased settlement. The critical zone then considers both the depth and eccentricity of the void. Practical solutions such as shotcrete or ground supports are implemented to reduce the risk of voids failure. For manmade underground structures, lining and reinforced concrete are used to build sustainable and durable structures.

Although design of tunnel lining as well as the effect of tunnelling on surface structures have been receiving great attention, there is no widely accepted design method. The mutual interaction between surface footings and tunnels could adversely affect their behaviours. Several investigations have been carried out numerically (see for example, Dessouki and Monforton, 1986 and Duddeck, 1987). Muir-Wood (1975) investigated circular tunnels installed in elastic ground. The bending moment was calculated after working out the pressure on the tunnel lining using the elliptical mode of failure. Dessouki and Monforton (1986) investigated the effect of soil failure on soil-steel structure systems by developing a plain strain finite element programme. It was found that numerical results were in good agreement with the experimental results of a model conduit. Some remarks for evaluating the safety of structures founded on grounds containing tunnels were developed based on the work by Duddeck (1987).

A number of experimental studies have been carried out to investigate the interaction between surface loads and behaviour of tunnels. Wang and Kaiser (1992) investigated the 
behaviour of tunnels in cohessionless soils including initial elastic response, intermediate yielding and ultimate collapse. A number of possible modes of behaviour have been identified. Their results indicated that the support pressure decreases with increasing ground displacements due to arching around opening. Gutter et al. (1990) carried out a number of centrifuge physical tests. The results of strain measurements in the tunnel lining were compared with those obtained numerically. They were in good agreement.

So far there is no comprehensive study considering the footing size as well as the tunnel size and its stiffness. In this paper, the interaction between surface strip footings and underground tunnels is investigated by conducting both physical tests (experiments) and numerical study using a numerical code "ABAQUS". Numerous experiments have been conducted. However, only a few presented in this paper. The results are presented in terms of the bearing capacity, footing deformation, tunnel deformation.

\section{PHYSICAL EXPERIMENTS}

\section{Experimental set-up and measurements}

A specially designed tank with internal dimensions of $1000 \mathrm{~mm}$ long, $200 \mathrm{~mm}$ wide and $600 \mathrm{~mm}$ deep is used in this investigation. Figure 1 shows a schematic diagram of the tank and loading system. The front wall of the tank is made of $10 \mathrm{~mm}$ thick SECURIT glass in order to allow visual observation and to withstand pressure. The back wall of the tank is made of steel strips with $100 \mathrm{~mm}$ height stiffened with steel angles. In order to change the tunnel dimensions, three strips with different heights are manufactured. The strips are designed in a way so that they can be bolted together in a way to facilitate the variation of tunnel depth and eccentricity between tunnel and footing centres. All strips are insulated with a rubber gasket to ensure a tight fit. A static load is applied using a loading device having 1:10 amplification.

Measurements of deformation and strain are carried out at the tunnel crown and both opposite sides (walls). All measurements are taken at the middle of the tunnel lining which is at $100 \mathrm{~mm}$ away from the edge of the tank. Three strain gauges were used to measure the strain in the tunnel lining at the same three locations on the tunnel lining but from outside. Strain gauges are mounted around the tunnel lining before insertion. They are connected with a strain indicator and initial readings are taken. Readings of deformation and strain were taken after each load increment.

\section{Materials}

A sandy soil with mean diameter of $0.52 \mathrm{~mm}$ is used in this investigation. The uniformity and curvature coefficients are 3.33 and 1.45 respectively. According to the BS 5950, this sand is classified as poorly graded silty sand. Standard compaction tests demonstrate that the maximum dry unit weight is $17.7 \mathrm{kN} / \mathrm{m}^{3}$ and can be achieved at moisture content of $12 \%$. The specific gravity of the used sand is 2.68 . Determination of the shear strength

parameters indicates that the used sand has an angle of internal friction of $40^{\circ}$ and 
apparent cohesion of $19.6 \mathrm{kPa}$ due to the presence of small percentage of silt and being wet. Galvanised steel with a thickness of $0.35 \mathrm{~mm}$ was used to form a tunnel lining.

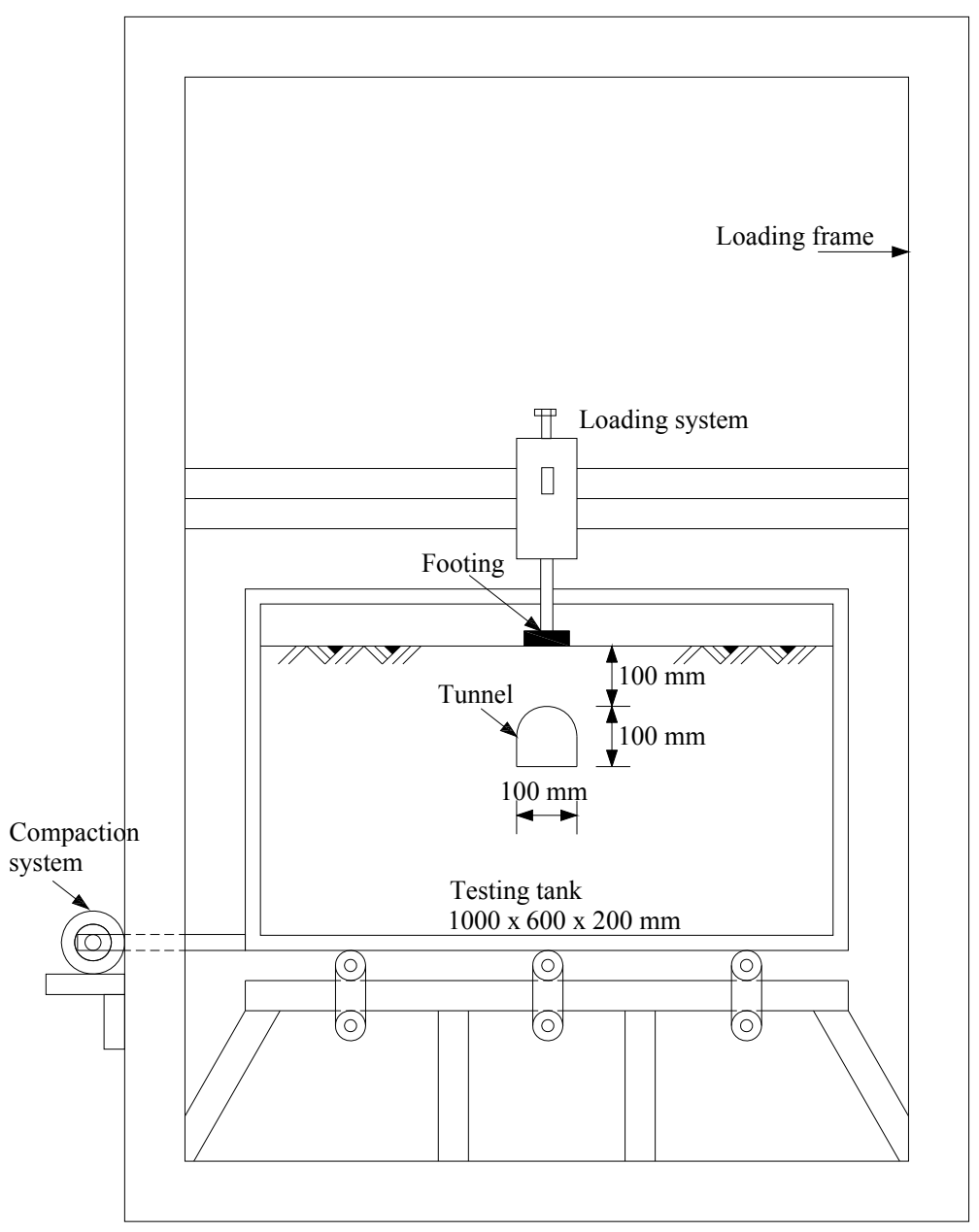

Figure 1: Schematic diagram of the experimental set-up

\section{Testing procedure}

The tank is filled up with the silty sand in $50 \mathrm{~mm}$ layers up to the level of the tunnel bed. Each layer is compacted manually. Then the tunnel lining is inserted from the back wall through the opening in the corresponding strip. The tunnel lining was kept in place using a wooden mould. The filling process is then continued as before till reaching the final predetermined level. Upon completion of tank filling, a second stage of compaction is started by placing a relatively heavy weight plate on top of the sand surface and shaking the whole tank for 75 seconds. After completing the compaction process, the sand surface was carefully levelled off and any extra sand is carefully removed. The footing was then placed on the centre of the tank and the loading device is attached to the footing. The load is applied in steps and continued until soil or tunnel failure occurs. 


\section{NUMERICAL SIMULATIONS}

In this paper, the non-linear finite element programme ABAQUS/Standard is used to undertake a two-dimensional (2D) plain strain analysis of the problem. Finite Element Method is employed for investigating the mutual effects between surface loads and tunnels. A numerical model is developed so that the considered problem can be investigated. An elaborated description of the models assigned for soil mass, tunnel structure and interaction between tunnel and surrounding soil is presented in this section. The soil material is modelled using the inelastic Mohr-Coulomb material model. The tunnel lining and footing are modelled as linear elastic materials.

\section{Material Model}

In this investigation the soil is considered as a dense silty sand material whose behaviour is modelled by an elasto-plastic Mohr-Coulomb material model. ABAQUS offers a Mohr-Coulomb model for modelling this class of material behaviour. This model uses the classical Mohr-Coulomb yield criterion including a straight line in the meridional plane and a six-sided polygon in the deviatoric plane. However, the ABAQUS Mohr-Coulomb model has a completely smooth flow potential instead of the classical hexagonal pyramid. The flow potential is a hyperbola in the meridional plane, and uses a smooth deviatoric section proposed by ABAQUS Theory Manual (2005). The values of Young's modulus and possion's ratio are assumed to be $7 \mathrm{MPa}$ and 0.3 respectively. Since the tunnel lining is made up of Galvanized steel, its behavior can be described as an elastic material. The galvanised steel lining has a density of $7800 \mathrm{Kg} / \mathrm{m}^{3}$, Young's modulus of $200 \mathrm{GPa}$, Poisson's ratio $(v)=0.3$ and rigidity of $2.55 \mathrm{X} 10-5 \mathrm{kNm}^{2}$.

\section{Soil-tunnel Interaction}

A precise simulation of the soil structure interface is vital for a successful modelling of the structural response. The soil-tunnel interaction is modelled using "Contact pair" models. A pure master-slave relationship is used to model contact between two surfaces ABAQUS Analysis Manual (2005). In this case the outer surface of the tunnel structure is modelled as "Master Surface" and the surrounding soil surface is modelled as "Slave Surface". In the ABAQUS calculations, a "finite sliding" formulation is used. Finite sliding allows for any arbitrary motion of the surfaces such as separation, sliding, rebound and rotation of the surfaces in contact, which should be needed in the analysis of the structure (Helwany, 2007).

\section{Model discretization}

A 3-node bilinear plane strain quadrilateral, reduced integration are used to model the sand medium with dimensions of $600 \mathrm{~mm}$ deep and $1000 \mathrm{~mm}$ length. In addition, a 4node bilinear plane strain quadrilateral, reduced integration elements are used to model the tunnel lining. The footing is modelled as steel, rigid and perfectly bonded to the soil surface. A footing with a width of $75 \mathrm{~mm}$ is investigated. Horizontal displacement 
boundary conditions are applied along the vertical edges by restraining the displacement in the horizontal direction and vertical displacement boundary conditions are applied along the base edge by restraining the displacement in the vertical direction. The overall geometric model is divided into three different regions representing the soil, tunnel and the rigid surface footing. A convergence study involving mesh refinement, appropriate selection of element types is initially performed; hence the optimum meshes are identified for the soil, tunnel and the rigid surface footing, as shown in Figure 2.

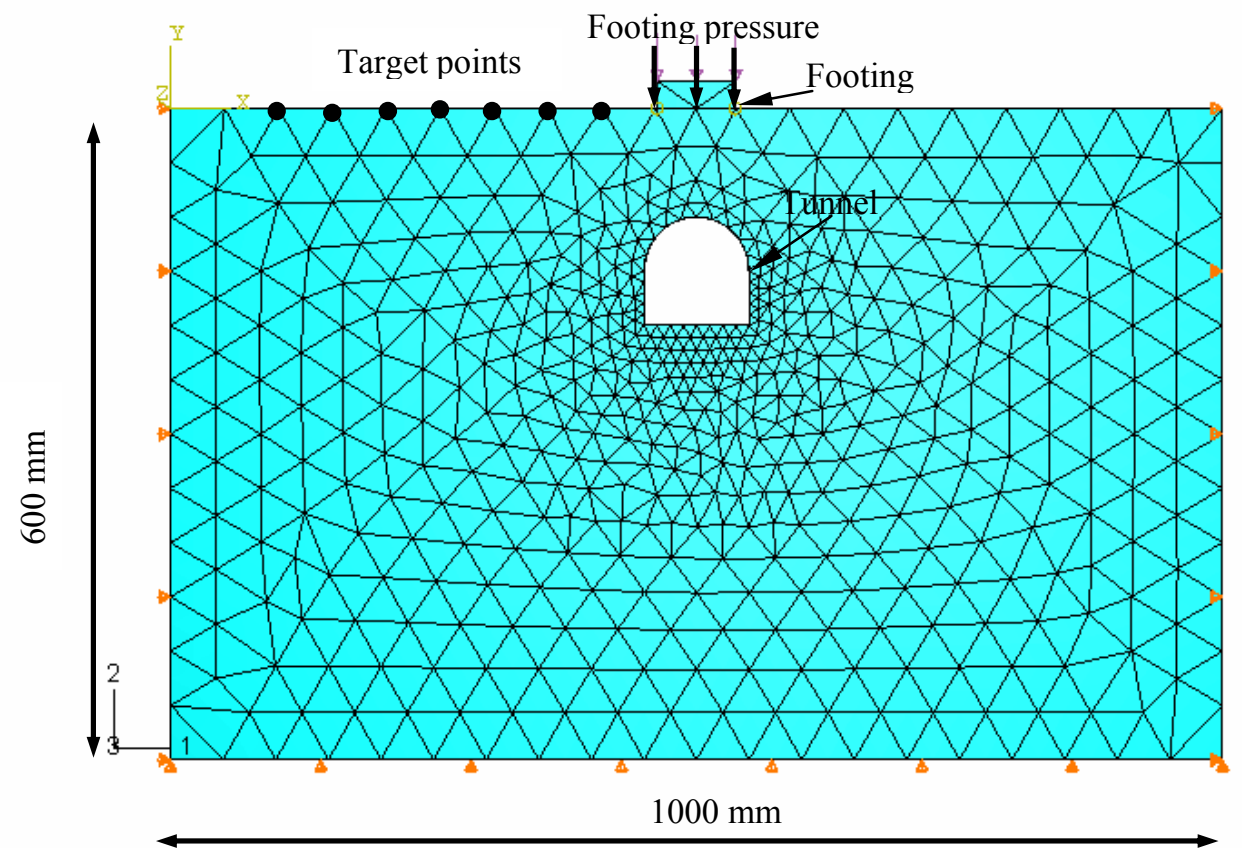

Figure 2: Finite element system modelling

It should be noted that automatic meshing is employed and therefore there might be slight difference in the elements around the central vertical axis. However, denser mesh is constructed in areas in which stresses are concentrated. An incremental external uniform pressure is applied on the footing. The pressure is varied between 0 to $500 \mathrm{kPa}$ on 100 $\mathrm{kPa}$ increments.

The tunnel lining is modelled as galvanized steel with constant thickness $(t)$ of $0.35 \mathrm{~mm}$. Both the internal height of the tunnel $(h)$ and tunnel width are kept at $100 \mathrm{~mm}$. The tunnel is buried in the sand at a depth $(d)$ of $100 \mathrm{~mm}$ and centred below the surface footing as shown in Figure 2. The entire footing-soil-tunnel system is represented by an assemblage of a finite number of 3 and 4-node bilinear plane strain quadrilateral, reduced integration elements. 


\section{Results and discussion}

Several model tests were performed to study the interaction between surface loading and underground tunnels. In this paper only the results for concentric tunnel that is located $100 \mathrm{~mm}$ underneath the surface footing are presented and discussed. Three different footing widths are investigated in this study. The tunnel width is $100 \mathrm{~mm}$. The tunnel deformation is measured at three different positions namely crown, side/wall 1 and side/wall 2. Since the footing is concentric with the tunnel, deformation of side 1 and side 2 are expected to be the same. Thus, only deformation of one side is presented.

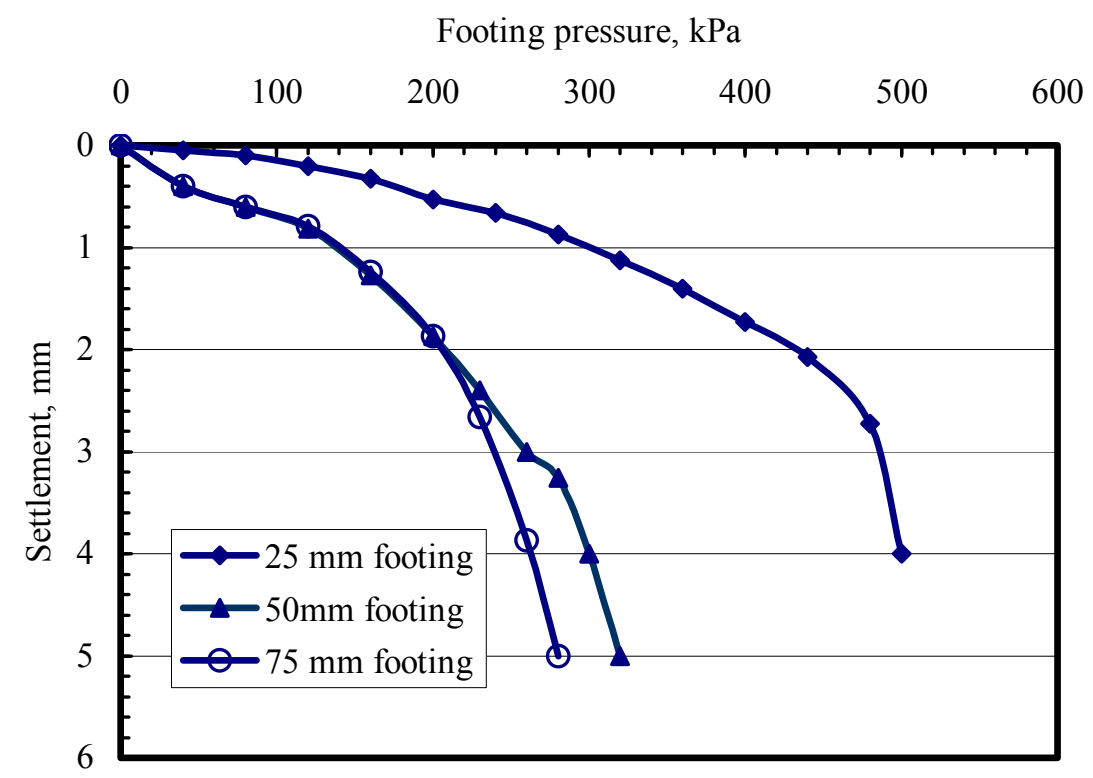

Figure 3: Settlement of surface footing against footing pressure

Figure 3 shows the results of footing settlement as a function of the footing pressure for different footing widths. The results demonstrate clearly that for the same tunnel width and depth, any increase in the footing width causes an increase in the footing settlement. The results demonstrate that the measured settlement for $50 \mathrm{~mm}$ and $75 \mathrm{~mm}$ footings is almost same up to a bearing pressure of $200 \mathrm{kPa}$. Then noticeable difference is clear for footing pressures above $200 \mathrm{kPa}$. Figure 3 shows that for the $25 \mathrm{~mm}$ footing, settlement increases slightly with footing pressure. Only a $0.5 \mathrm{~mm}$ settlement is recorded at a pressure of $200 \mathrm{kPa}, 2.8 \mathrm{~mm}$ at $500 \mathrm{kPa}$ and failure occurs at $520 \mathrm{kPa}$. For larger footings, the footings experienced $\sim 1.0 \mathrm{~mm}$ settlement at a pressure of $140 \mathrm{kPa}$. Then sharp increase in the rate of settlement occurred with any further increase in the footing pressure. The ultimate pressure is $310 \mathrm{kPa}$ and $260 \mathrm{kPa}$ for footing width of $50 \mathrm{~mm}$ and $75 \mathrm{~mm}$ respectively

The influence of footing width on crown deflection for the same tunnel width and depth is shown in Figure 4. It can be seen that crown deflection increases as the footing pressure increases. For $50 \mathrm{~mm}$ and $75 \mathrm{~mm}$ footings less crown deflection is recorded for $75 \mathrm{~mm}$ footing up to a footing pressure of $200 \mathrm{kPa}$. The slightly smaller crown deflection 
for the largest footing at low footing pressure could be due to a stabilization effect of the pressure transferred to the bottom of the tunnel arch. This transferred pressure helps in reducing the deflection of the crown. Generally, crown deflection follows qualitatively the same trend as the footing settlement. This indicates that the footing width would cause an increase in the load transfer to the tunnel lining which in turn causes an increase in the crown deflection. For the smallest footing, failure of tunnel lining was not observed. However, the footing failure was due primarily to the soil cover underneath the footing and above the tunnel.

The results of footing settlement and deflection of tunnel crown suggest that stresses are concentrated in a zone that is directly beneath the surface footing. This zone is called the influenced zone and is strongly related to the footing width. Thus for smaller footings the bearing pressure settlement relation would be controlled primarily by the characteristics of the direct soil underneath the footing with minimal interaction with tunnel since the tunnel depth is four times the footing width. As the footing width increases, stress is concentrated in a zone that is directly above the tunnel crown. This would increase the crown deformation. However, with further enlargement of the influenced zone as a result of relatively large footings, stresses are supporting the tunnel sides which would result in a reduction in the measured tunnel crown deflection. In this case, there is a mutual influence on the behaviour of both footing and tunnel. With further increase in footing width, more stresses are concentrated on the tunnel arch leading to large settlement as well as reduced ultimate bearing pressure.

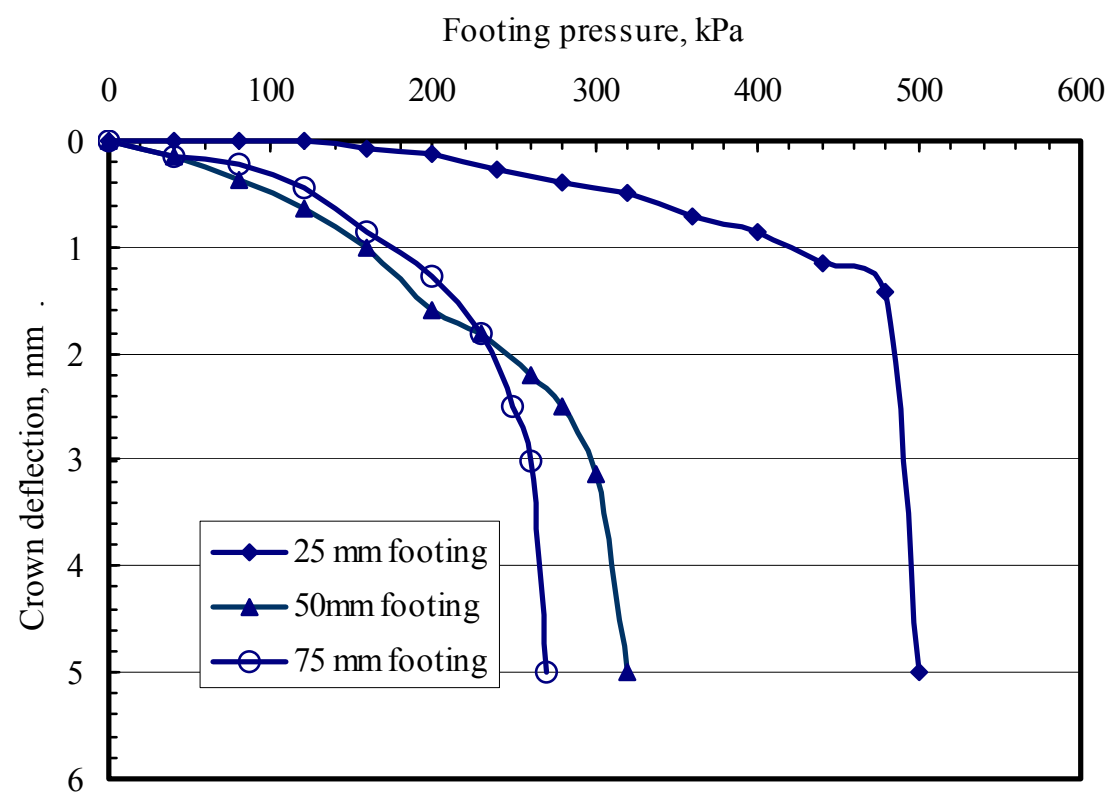

Figure 4: Crown deflection against the footing pressure for different footing widths 


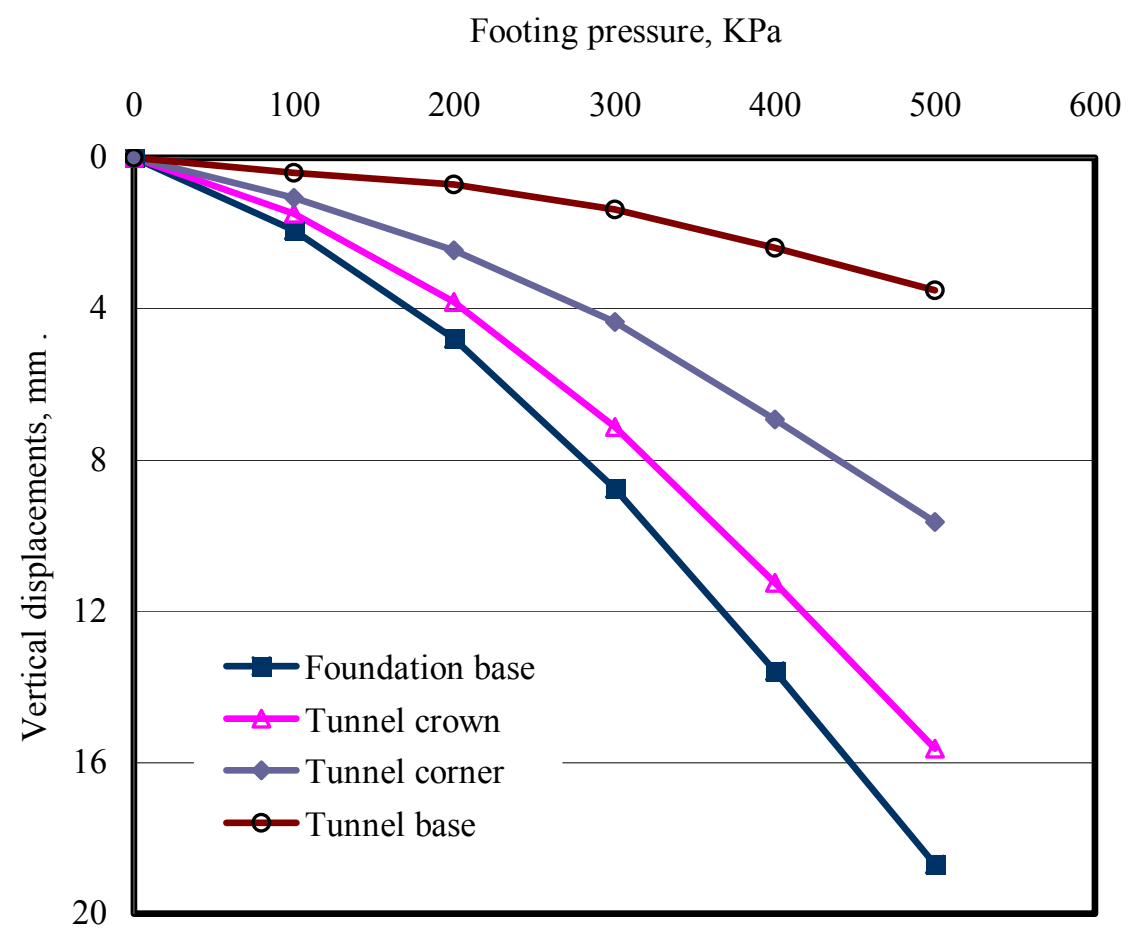

Figure 5: Numerically obtained results for settlement

Results of footing settlement and deformation of tunnel obtained numerically are presented in Figures 5. It can be seen that footing settlement increases with the increase in the applied footing pressure with no definite failure for the footing-tunnel system. Settlement reaches the value of $18.5 \mathrm{~mm}$ when the applied footing pressure is $500 \mathrm{kPa}$. Comparing data for the footing settlement obtained numerically with actual records of settlement from physical testing demonstrates that numerical simulation does not capture the failure of the footing-tunnel system at $280 \mathrm{kPa}$ nor its results are close to the measured. Interesting results for the tunnel base deformation show that the corner of the tunnel always suffers a settlement whereas the deformation of the tunnel base centre is upward.

Deformation of the ground surface is also obtained numerically at various applied footing pressure and shown in Figure 6. The data presented in Figure 6 depict that most of the deformation occurs underneath the footing which in this investigation is located $100 \mathrm{~mm}$ above and concentrically with the tunnel. The results clearly show that no heave would be experienced which is contradictory to the observation in the laboratory. This could be due to limited capabilities of the model assigned to simulate the soil behaviour. Results of the vertical stress distribution in the soil medium is presented in Figure 7 for an applied footing pressure of $500 \mathrm{kPa}$. These results demonstrate clearly that for the current problem, arching is working on resulting in the transfer of portion of the developed vertical stress to the sides of the tunnel supporting the tunnel arch. These results explain the measured crown deflection presented in Figure 5. Most of the stresses are concentrated on the tunnel arch. Stresses are reduced towards the tunnel base. Higher 
stresses applied on the sides of tunnel would be offering a stabilising effect reducing the deformation of tunnel crown.

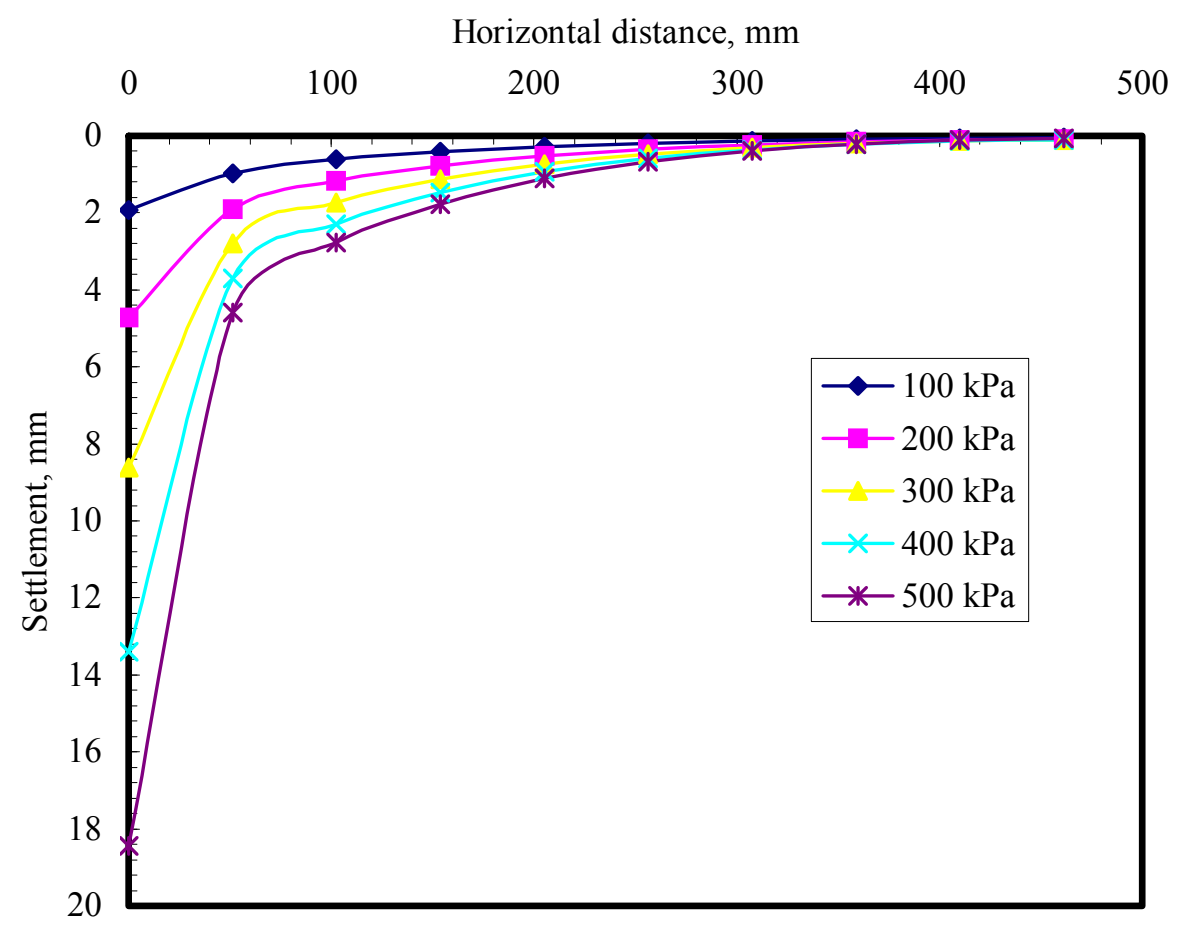

Figure 6: Numerical results for ground settlement

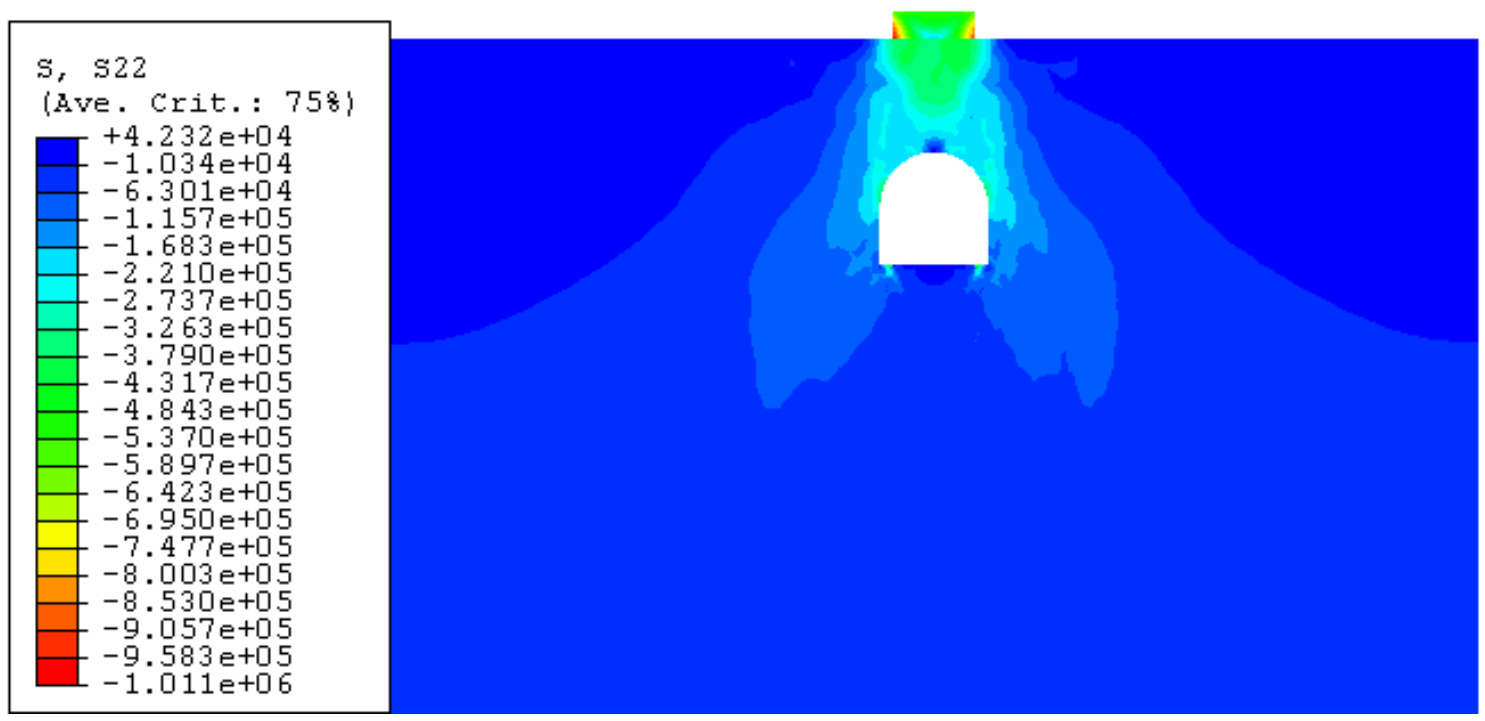

Figure 7: Stress distribution for an applied footing pressure of $500 \mathrm{kPa}$

The preliminary results of the numerical simulations seem slightly rough in capturing all features of the behaviour of footing-tunnel system. Further investigations are underway to 
determine appropriate and meaningful soil parameters as well as to the use of more sophisticated soil model such as Draker-Prager's model.

\section{Conclusions}

In this paper an investigation is carried out into the interaction between footing and shallow tunnel buried in silty sand. A number of physical tests are conducted in which deformation of footing and tunnel is measured. In addition numerical simulations are undertaken using numerical code "ABAQUS". Soil and Galvanised steel are modelled as an elastic-plastic and elastic materials respectively. The following are some of the main conclusions that can be made out of the investigation:

- For small footings, it is most likely that the behaviour of footings will be controlled by the soil behaviour underneath the footings rather than by the tunnel.

- For medium and large footings, it is likely that there will be a mutual interaction between the surface footings and buried tunnels resulting in a relatively increased deformation of both structures.

- For large footings, stresses might develop on the sides of the tunnel resulting in a stabilising pressure leading to a slight reduction on the tunnel and footing settlements. However, as the applied footing pressure increases, large settlement occurs.

- Results of the numerical simulations show that footing settlement increases with the applied footing pressure with no obvious failure.

- Numerically obtained results for stress distribution in the soil clearly explain the behaviour of the footing and tunnel system.

- Numerically obtained deformations are much greater than those obtained experimentally, raising question marks concerning the model assigned for capturing the behaviour of the soil and steel materials. Further investigations are underway to determine accurate values for the soil parameters and to use a more sophisticated model for the soil.

\section{References}

ABAQUS Analysis Manual (2005), ABAQUS Analysis User's Manual, Version 6.5, Published by Hibbitt, Karlsson and Sorensen Inc. USA.

ABAQUS Theory Manual (2005), Version 6.5. Published by Hibbitt, Karlsson And Sorensen Inc. USA.

Baus, R.L. and Wang, M.C. (1983). Bearing Capacity of strip footing above void Journal of Soil Mechanics and Foundation Engineering, ASCE, Vol 109(1) pp 1-13.

Dessouki, A.K. and Monforton, G.R. (1986). Effect of soil failure on soil-steel structure. Journal of Geotechnical Engineering, ASCE, Vol112 (5), pp 522-536.

Duddeck, H. (1987). Generl Approaches to the design of underground openings. VI Australian Tunnelling Conference, Melbourne, pp 159-172.

Gutter, U., Piepenbreier, G. and Jessberger, H.L. (1990). The effect of foundation loads on the design of tunnel lining. Proceedings of the $12^{\text {th }}$ International Conference on Soil Mechanics and Foundation Engineering, pp 921-924. 
Helwany, S. (2007), Applied soil mechanics with ABAQUS`applications. Hoboken, New Jersey: John Wiley \& Sons, INC

Meyerhof, G.G. (1963). Some recent research on the bearing capacity of foundations. Canadian Geotechnical Journal, Vol 1(1).

Muir-Wood, A.M. (1975). The behaviour of circular tunnel in elastic ground. Journal of Geotechnique, Vol. 25 (1), pp 115-127.

Terzaghi, K. (1943). Theoretical Soil Mechanics. John Wiley \& Sons, New York.

Wong, R.C.K. and Kaiser, P.K. Performance assessment of tunnels in cohesionless soils. Journal of Geotechnical and Geoenvironmental Engineering, ASCE, Vol 117 (12), pp 1180-1901.

Wood, L.A. and Larnach, W.J. (1984). The behaviour of footings located above voids. Proceedings of the Eleventh International Conference on Soil Mechanics and Foundation Engineering, Vol. 4, pp 2273-2276. 